\title{
Emphysematous Lung Sealant Therapy Attenuates the Chronic Inflammatory State of Advanced Emphysema
}

Kramer MR $^{1^{*}}$, Fruchter $\mathrm{O}^{2}$, Ralf Eberhardt ${ }^{2}$, Valipour $\mathrm{A}^{3}$, Assadi $\mathrm{S}^{3}$, Bonnet $\mathbf{R}^{4}$, Maeder ${ }^{4}$, Kempa $\mathrm{A}^{5}$, Stanzel $\mathrm{F}^{5}$, Ingenito EP and Felix Herth ${ }^{6}$

${ }^{1}$ Pulmonary Institute Rabin Medical Center, Beilinson Hospital, Petach Tikva, Israel

2Pneumology and Critical Care Medicine, Thoraxklinik, Heidelberg University, Germany

${ }^{3}$ Pulmonary and Critical Care Medicine, Otto Wagner Hospital, Vienna, Austria

${ }^{4}$ Bad Berka Medical Center, Department of Pneumology, Bad Berka, Germany

${ }^{5}$ Lungenklinik Hemer, Department of Pulmonary Medicine, Hemer, Germany

${ }^{6}$ Aeris Therapeutics, Medical Affairs Department, Woburn MA, USA

\begin{abstract}
Background: Patients with advanced emphysema often have elevated markers of systemic inflammation, a phenotype associated with greater clinical compromise and increased mortality. We hypothesized that endoscopic lung volume reduction using emphysematous lung sealant therapy might attenuate the state of inflammation in patients with advanced emphysema by eliminating damaged tissue; similar to what has previously been reported following lung volume reduction surgery.
\end{abstract}

Methods: To explore this hypothesis, serum C-reactive protein (CRP) was measured at baseline, 12, 24 and 48 weeks following ELVR therapy with Emphysematous Lung Sealant (ELS) at 5 centers participating in clinical trial NCT00884962 (www.clinicaltrials.gov). Results were correlated with post-treatment changes in BODE Index, systemic white cell count, and vital status out to 3 years.

Results: Baseline CRP for the 29 patient cohort was elevated $(5.3 \pm 8.7 \mathrm{mg} / \mathrm{L})$ compared to normal. Sixteen (16) of 29 patients $(55 \%)$ had serum levels above the "high risk threshold" ( $3 \mathrm{mg} / \mathrm{L})$. ELS therapy was associated with a transient increase in CRP followed by a progressive decline. At 48 weeks, the CRP level of the cohort had decreased to $2.2 \pm 3.2 \mathrm{mg} / \mathrm{L}$ ( $p=0.041$ ); only 9 of $29(31 \%)$ showed persistently elevation. 3 years post treatment, 28 of 29 patients were alive (3.4\% mortality), while 24 and 36 month mortality for this cohort predicted from baseline BODE Index was $12 \%$ and $35 \%$ respectively.

Conclusions: ELS therapy reduces chronic inflammation in patients with advanced emphysema, manifest as a reduction in CRP. Model predictions suggest this is associated with a reduction in long term mortality risk. Larger prospective studies are needed to further evaluate this question.

Keywords: Endoscopic lung volume reduction; Lung sealant therapy; CRP levels in emphysema

\section{Introduction}

Recent studies have shown that a subgroup of patients with advanced emphysema experience active chronic inflammation characterized by an elevation in circulating inflammatory markers [13]. Increased serum levels of interleukin 6 (IL-6), IL-8, IL-16, tumor necrosis factor alpha (TNFa), fibrinogen, and C-reactive protein (CRP) have been reported, and imbalances between pro-inflammatory and pro-remodeling cytokines and growth factors demonstrated. This "inflammatory" phenotype is associated with more frequent exacerbations, a more rapid decline in lung function, and higher mortality than in patients without elevated inflammatory biomarkers $[1,3,4]$. While anti-inflammatory medications, including inhaled corticosteroids and phosphodiesterase- 4 inhibitors have been shown to reduce the frequency of COPD exacerbations in susceptible individuals, they have not been shown to alter the natural progression of disease in GOLD Group D patients, and their clinical and biological effects on chronic systemic inflammation have not been characterized [5].

Lung Volume Reduction Surgery (LVRS) has recently been shown to reduce circulating mediators of inflammation in patients with advanced emphysema [6,7]. Independent studies have confirmed that LVRS reduces exacerbation rates, improves functional outcomes, and reduces mortality is subgroups of emphysema patients [8,9]. While a direct link between the clinical benefits of LVRS and its purported anti-inflammatory effects has not been shown, some of the benefits associated with LVRS may theoretically occur via down-regulation of chronic inflammation.
In clinical practice LVRS is now rarely performed, having been supplanted by endoscopic techniques. While Endoscopic Lung Volume Reduction (ELVR) can indeed replicate the physiological effects of LVRS, the effects of ELVR on the inflammatory state of emphysema have been studied.

We hypothesized that endoscopic lung volume reduction therapy using Emphysematous Lung Sealant (ELS) might replicate the biologic effects of LVRS on chronic inflammation in emphysema, since like surgery, it leads to the elimination of the targeted tissue. To explore this hypothesis we assessed CRP levels pre- and post- treatment out to 48 weeks, and correlated results with observed and predicted long term mortality projected from baseline BODE Index.

\section{Materials and Methods}

A retrospective analysis of clinical and biomarker data was

*Corresponding author: Mordechai R Kramer MD, Director of Pulmonary Institute and Chief of Lung Transplantation, Rabin Medical Center, Beilinson Hospital, Petach Tikva, Israel 49100, Tel: +1-617-833-853; E-mail: kremerm@clalit.org.il orenfr@clalit.org.il

Received November 05, 2013; Accepted December 09, 2013; Published December 11, 2013

Citation: Kramer MR, Fruchter O, Eberhardt R, Valipour A, Assadi S, et al. (2013) Emphysematous Lung Sealant Therapy Attenuates the Chronic Inflammatory State of Advanced Emphysema. J Pulm Respir Med 3: 163. doi:10.4172/2161 105X.1000163

Copyright: (c 2013 Kramer MR, et al. This is an open-access article distributed under the terms of the Creative Commons Attribution License, which permits unrestricted use, distribution, and reproduction in any medium, provided the original author and source are credited. 
performed on 29 patients with advanced (GOLD Group D patients, Grade III and IV airflow obstruction) emphysema enrolled in clinical trial NCT \#00884962 (www.clinicaltrials.gov). This cohort includes all patients who had baseline and follow-up serum CRP levels measured during the course of the study. The study was conducted in accordance with GCP guidelines, and all protocols were approved by the appropriate regional regulatory authorities and hospital ethics committees. Patients were enrolled at 3 sites in Germany, 1 in Austria, and 1 in Israel. Complete data sets were available for all 29 patients from baseline through week 48 . Vital status was determined for all 29 patients out to 3 years post treatment.

A complete list of study inclusion/exclusion criteria is presented on www.clinicaltrials.gov. Qualifying patients were required to have GOLD Group D emphysema despite optimal medical therapy, and be without co-morbid conditions that might affect their ability to respond to ELVR treatment or complete long-term follow-up. Patients with alpha-1 antitrypsin deficiency were excluded. The study cohort included 11 patients with upper lobe predominant heterogeneous disease (ULP), 2 patients with non-upper lobe predominant heterogeneous disease (NULP), and 16 patients with homogeneous disease (Ho). All patients received ELS treatment at 4 sub segments administered in divided doses over 2 treatment sessions 12 weeks apart, with 2 sub segments treated during each session. Target sites were selected based on pretreatment CT imaging, with the most severely damage areas of lung receiving treatment.

Physiological, functional, and biomarker assessments were performed at 12, 24 and 48 weeks post treatment. Beyond week 48, vital status was recorded yearly out to 3 years.

Responses to ELS therapy were expressed as change from pretreatment baseline for all outcome measures. Change from baseline was assessed by paired $t$-test for continuous variables (CRP values, white cell count, fibrinogen level, FEV1, SGRQ, and 6 MWT distance) and by Wilcoxon rank sum test for nominal variables (BODE Index, MRCD scores). Comparisons of categorical data were performed by Pearson's
Chi squared test. Statistical significance was assessed by 2 -tailed testing based on $\mathrm{P}$ values corrected for multiple comparisons using the method of Bonferroni where appropriate (i.e. $\mathrm{P}<0.05 / \mathrm{n}$ where $\mathrm{n}$ is number of comparisons performed).

\section{Results}

Demographic and pre-treatment physiological characteristics for the 29 patient cohorts are summarized in Table 1. These baseline characteristics are representative of patients with advanced emphysema. All patients had GOLD Group D disease with severe or very severe airflow obstruction and persistent symptoms despite optimal medical therapy.

Sixteen (16) of 29 patients had baseline CRP levels considered to be in the elevated or "high-risk" range (i.e. $\geq 3 \mathrm{mg} / \mathrm{L}$ ) [10]. Table 2 summarizes pre-treatment baseline characteristics of this "high-risk" group relative to the other 13 patients in the study whose baseline CRP values were in the "low-risk" range. The 2 groups were similar in age and gender distribution, medication use throughout the study, oxygen dependency, had similar baseline pulmonary function, exercise capacity, (i.e. \%predicted FEV1, \% predicted RV, \%predicted DLco, and 6 minute walk test distance) and BODE Index values, but had significantly different smoking histories and CRP levels ("high-risk" group pk $\mathrm{yrs}=59.1 \pm 30.6$ vs. "low risk" group $=36.8 \pm 19.4, \mathrm{P}=0.022$; "high risk" group $\mathrm{CRP}=9.2 \pm 10.2 \mathrm{mg} / \mathrm{L}, \mathrm{n}=16$ vs. "low risk" group $\mathrm{CRP}=0.6 \pm 0.8$ $\mathrm{mg} / \mathrm{L}, \mathrm{n}=13, \mathrm{P}=0.003)$. There were no significant differences between the groups with respect to number of exacerbations during the year preceding study enrollment, use of inhaled corticosteroids, or use of oral phosphodiesterase inhibitors. Baseline CRP levels failed to correlate with post-bronchodilator baseline \%predicted FEV 1 (Pearson correlation $\mathrm{r}=0.187, \mathrm{n}=29, \mathrm{p}>0.1$ ), baseline \% predicted RV (Pearson correlation $\mathrm{r}=0.163, \mathrm{n}=29, \mathrm{p}>0.1$ ), baseline circulating WBC (Pearson correlation $\mathrm{r}=0.079, \mathrm{n}=29, \mathrm{p}>0.1$ ), or baseline BODE Index (Spearman correlation $\mathrm{r}=0.117, \mathrm{n}=29, \mathrm{P}>0.1$ ) Thus, among patients enrolled in this trial, baseline CRP appeared to uniquely identify a cohort of GOLD Group D patients with a pro-inflammatory state not represented by alternate clinical biomarkers.

\begin{tabular}{|c|c|c|c|c|c|c|}
\hline DEMOGRAPHICS and $\mathrm{O2}$ & Age & Fraction males & pk yr & BMI & Exac Prev Yr & Oxygen Use \\
\hline$n=29$ & $60.7(8.3)^{\star}$ & 0.66 & $49.2(28.2)^{*}$ & $25.4(3.5)^{*}$ & $0.57(0.66)^{*}$ & $10 / 29$ \\
\hline \multirow{2}{*}{ MEDICATION USE } & SABA & LABA & SAAC & LAAC & ICS & Theophylline \\
\hline & $2 / 29$ & $26 / 29$ & $2 / 29$ & $23 / 29$ & $23 / 29$ & $3 / 29$ \\
\hline \multirow{2}{*}{ BASELINE PHYSIOLOGY } & Baseline RV & RV/TLC & FEV1 & DLco & 6 MWT & MRCD \\
\hline & $224.2(66.6)^{*}$ & $0.61(0.12)^{*}$ & $33.0(8.4)^{*}$ & $349(119)^{*}$ & $349(119)^{*}$ & $3.0(0.6)^{*}$ \\
\hline
\end{tabular}

"Mean values presented with standard deviation in parentheses.

Table 1: Demographics and baseline medical and physiological characteristics of 29 patient cohort with biomarker data.

\begin{tabular}{|c|c|c|c|c|c|c|c|}
\hline DEMOGRAPHICS and $\mathrm{O} 2$ & Age & M/F ratio & pk yr & BMI & Exac Prev Yr & \multicolumn{2}{|c|}{ Oxygen use } \\
\hline High Risk ( $n=16)$ & $60.2(9.5)$ & $10 / 6$ & $59.1(30.6)$ & $26.1(3.5)$ & $0.50(0.61)$ & \multicolumn{2}{|c|}{$5 / 16$} \\
\hline Low Risk $(n=13)$ & $61.3(7.0)$ & $9 / 4$ & $36.8(19.4)$ & $24.5(3.3)$ & $0.77(0.72)$ & \multicolumn{2}{|c|}{$5 / 13$} \\
\hline$p$ & 0.76 & & 0.02 & 0.35 & 0.14 & & \\
\hline MEDICATION USE & SABA & LABA & SAAC & LAAC & ICS & \multicolumn{2}{|c|}{ Phosphodisterase Inhibitor } \\
\hline High Risk (n=16) & $12.5 \%$ & $81.3 \%$ & $0,00 \%$ & $87.5 \%$ & $68.8 \%$ & \multicolumn{2}{|c|}{$6.3 \%$} \\
\hline Low Risk (n=13) & $23.1 \%$ & $100.00 \%$ & $15.3 \%$ & $69.2 \%$ & $92.3 \%$ & \multicolumn{2}{|c|}{$15.3 \%$} \\
\hline \multicolumn{8}{|l|}{$\mathrm{p}$} \\
\hline Baseline physiology & Baseline RV (\% pred) & RV/TLC & FEV1 (\% pred) & DLco (\% pred) & 6 MWT (meters) & MRCD (units) & BODE index \\
\hline High Risk (n=16) & $216.5(66.3)$ & $0.61(0.11)$ & $35.1(9.1)$ & $40.8(18.4)$ & $345(123)$ & $3.0(0.5)$ & $5.4(1.6)$ \\
\hline Low Risk $(n=13)$ & $233.6(68.3)$ & $0.61(0.13)$ & $30.3(6.7)$ & $37.8(19.0)$ & $353(120)$ & $3.0(0.5)$ & $5.7(1.2)$ \\
\hline$p$ & 0.46 & 0.95 & 0.09 & 0.69 & 0.88 & 1.0 & 0.52 \\
\hline
\end{tabular}

Table 2: Comparison of baseline demographics, medical use, and physiology of "High Risk" and "Low Risk" subgroups based on pre-treatment CRP values. 
Citation: Kramer MR, Fruchter O, Eberhardt R, Valipour A, Assadi S, et al. (2013) Emphysematous Lung Sealant Therapy Attenuates the Chronic Inflammatory State of Advanced Emphysema. J Pulm Respir Med 3: 163. doi:10.4172/2161-105X.1000163

Serum CRP levels during the course of follow-up out to 48 weeks are summarized in Figure 1. At 12 weeks post treatment, CRP levels were increased compared to baseline, an expected finding reflecting the post treatment inflammatory reaction that accompanies ELS therapy [11]. Elevations in CRP levels at this time point were observed in both "high risk" and "low risk" populations. By week 24, CRP levels in the "low risk" group had declined towards baseline, but remained elevated compared to pre-treatment, although differences from baseline were not statistically significant. In the "high-risk" group, CRP levels had decreased below pre-treatment baseline. By week 48, levels in both groups had decreased further, and mean values had settled below the "high-risk level" of $3 \mathrm{mg} / \mathrm{L}$. CRP levels in the "high risk" subgroup were significantly reduced compared to pre-treatment baseline $(9.2 \pm 10.2$ $\mathrm{mg} / \mathrm{L}$ vs. $2.5 \pm 2.5 \mathrm{mg} / \mathrm{L}, \mathrm{n}=16, \mathrm{p}=0.001$ ), while those in the "low risk" subgroup were not significantly different from pre-treatment baseline $(0.6 \pm 0.8 \mathrm{mg} / \mathrm{L}$ vs. $1.9 \pm 3.8 \mathrm{mg} / \mathrm{L}, \mathrm{n}=13, \mathrm{p}=0.441)$. Medication usage during the course of the study, and exacerbation frequencies in "high risk" ( $1.34 \pm 0.77$ events/yr) and "low risk" $(1.55 \pm 0.84$ events/yr $)$ groups were not significantly different $(\mathrm{p}=0.77)$.

Circulating white blood cell counts (WBCs) throughout the course of follow-up are summarized in Figure 2 and show a profile distinct from that of CRP. WBC levels were equivalent at baseline in "high risk" and "low risk" populations, and did not change in relation to ELS therapy during the course of the study at the designated follow-up time points. BODE Index, summarized in Figure 3, decreased in response to ELS therapy by the 12 week time point, and remained below pre-treatment baseline in both low and high risk subgroups through week 48 .

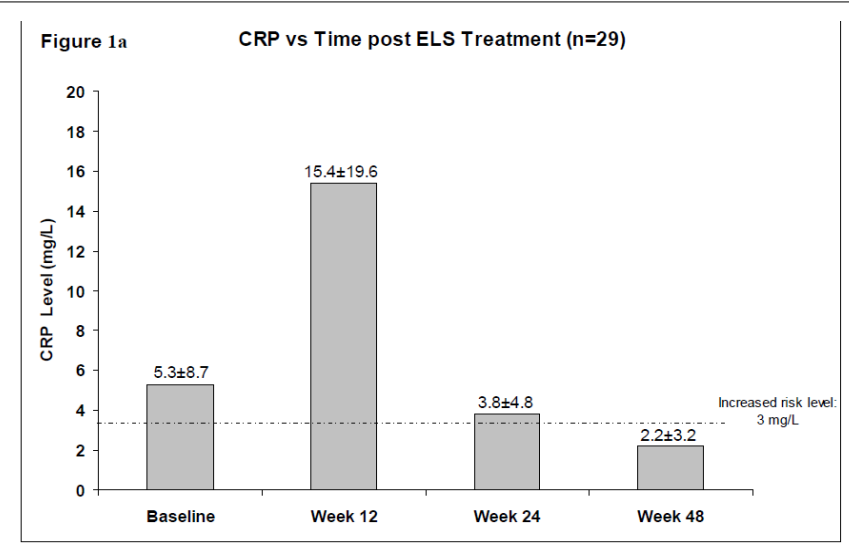

Figure 1b CRP levels in "High Risk" (n=16) and "Low Risk" (n=13) Subgroups Pre and Post ELS Treatment

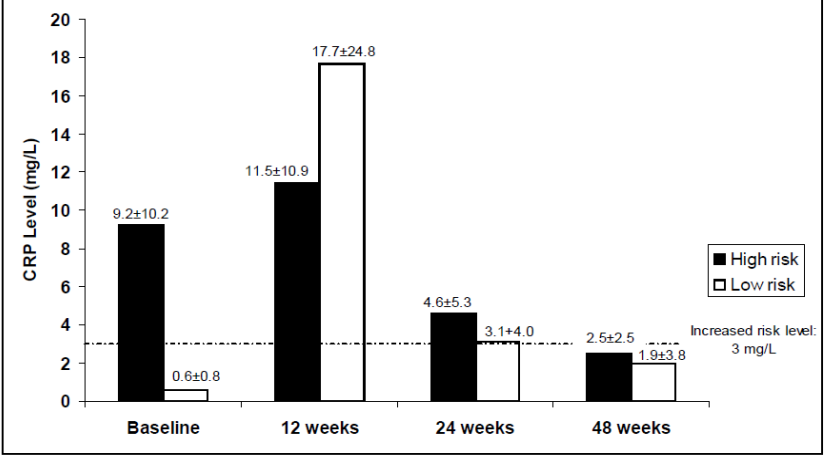

Figure 1: (a: upper) shows serum CRP levels as a function of time out to 48 weeks post ELS therapy in patients with GOLD Group D Emphysema for all patients in the study. (b: lower) shows serum CRP levels for "High Risk" and "Low Risk" subgroups separately.
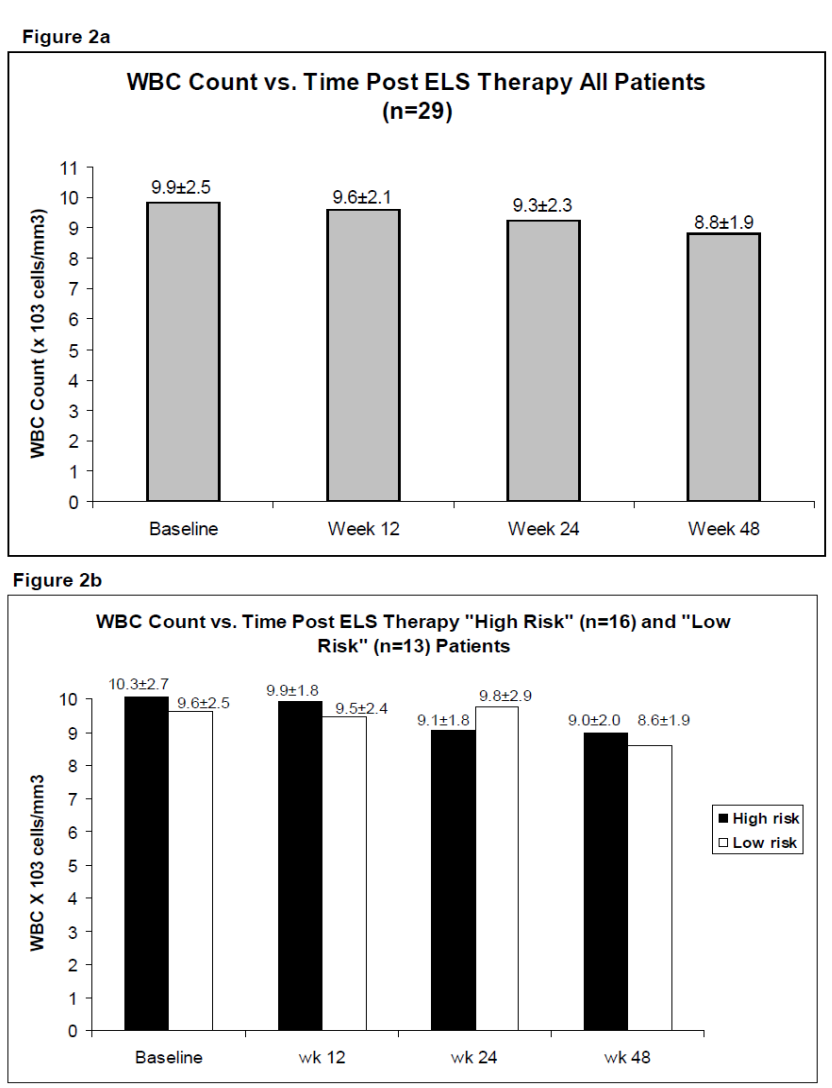

Figure 2: Circulating WBCs as a function of time following ELS therapy out to 48 weeks for all study patients ((a), upper panel), and for "High Risk" and "Low Risk" subgroups separately ((b), lower panel).

Baseline BODE Index values were used to predict mortality for the 29 patient cohort, and "high risk" $(\mathrm{n}=16)$ and "low risk" $(\mathrm{n}=13)$ subgroups out to 36 months. Results are presented in Figure 4, along with the actual vital status of study patients. Mortality among the 29 patient cohorts at 36 months following ELS treatment is substantially lower than predicted mortality based on baseline BODE Index values.

\section{Discussion}

Chronic inflammation associated with inhalation of foreign particulates is thought to play a central role in the pathobiology of advanced emphysema [12-15]. While inflammation in active smokers is expected, inflammation has also been demonstrated in the tissues of patients with advanced Group D (GOLD Stage III and IV) emphysema who have abstained from smoking for years $[16,17]$. These findings suggest that once established, the inflammatory state in certain patients with emphysema persists, and contributes to progressive clinical deterioration. There is evidence from several studies indicating that persistent inflammation, assessed in terms of elevated inflammatory biomarkers, is associated with premature mortality in emphysema, similar to what is observed in patients with coronary heart disease (CHD). Since CHD is a major cause of death in emphysema patients, it is possible that chronic inflammation among emphysema patients contributes to mortality by increasing risk of coronary death [18-20].

LVRS has been shown to alter the inflammatory milieu of patients with advanced emphysema, and it has been suggested that these effects may be due to resection of inflamed, damaged tissue that fuels the inflammatory process6. LVRS has now been largely replaced by endoscopic approaches which attempt to the replicate the physiological 


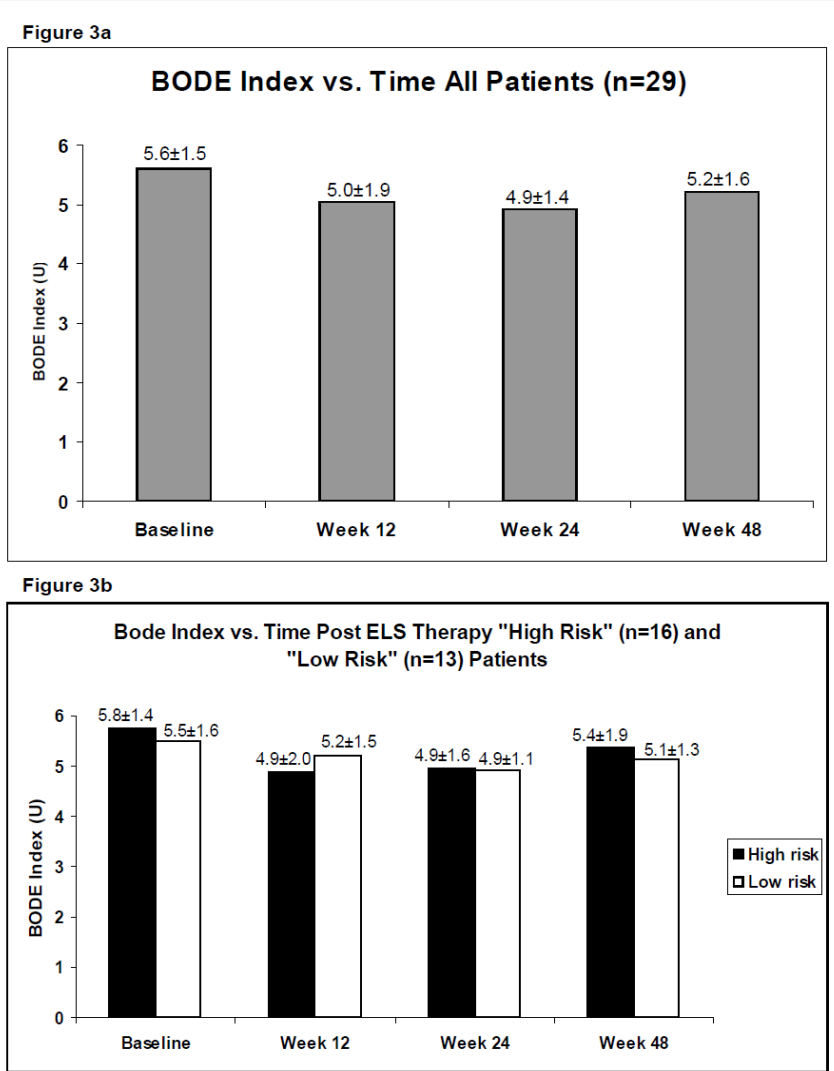

Figure 3: BODE Index as a Function of Time Following ELS Therapy Out to 48 Weeks Post Treatment for all study patients ((a), upper panel) and for "High Risk" and "Low Risk" patients separately ((b), lower panel).

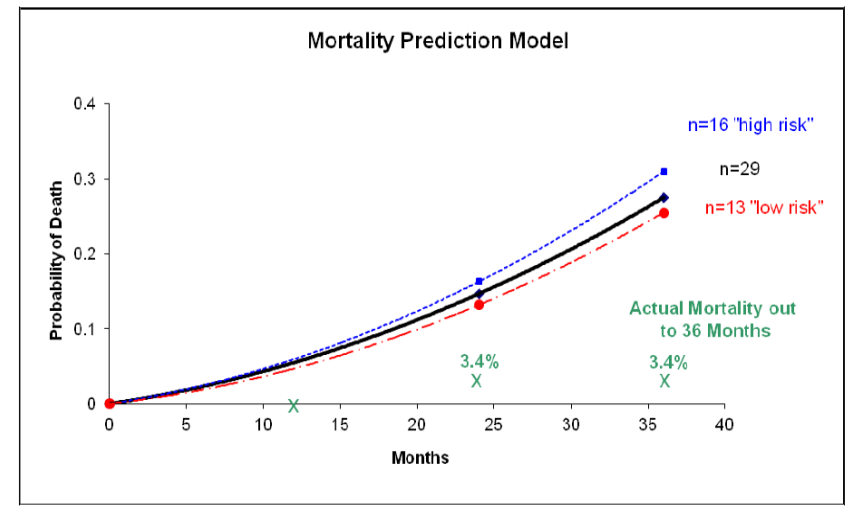

Figure 4: Predicted mortality for the 29 patient cohort, "High Risk" subgroup, and "Low Risk" based on baseline bode index relative to actual vital status.

effects of LVRS by collapsing rather than resecting damaged lung tissue. Results presented here indicate that collapse of damaged lung using ELS therapy may have beneficial effects on the state of chronic inflammation in advanced emphysema similar to surgical resection. CT imaging of patients receiving ELS therapy indicate that over time, treatment is associated with scarring of the targeted regions. Treatment of hyperinflated damaged areas, which results in permanent collapse, loss of blood flow, and subsequent scarring may be functionally equivalent to tissue resection with respect to eliminating zones of active inflammation. Thus ELS therapy may replicate both the physiological as well as biological effects of lung volume reduction surgery.
Simple "elimination" of inflamed tissue may not fully account for the benefits of ELS therapy or LVRS on the inflammatory state of emphysema, however. Both interventions are associated with post treatment inflammation: with LVRS this relates to the post surgical condition, while with ELS it relates to the post treatment inflammatory response associated with treatment, although it is difficult to quantify the extent to which the 2 procedures differ in this respect $[11,21]$. In both instances, systemic inflammation is likely associated with a post treatment Compensatory Anti-Inflammatory Response (CARS). CARS-linked cytokines have indeed been measured in emphysema patients following LVRS therapy, and may act to down-regulate subsequent inflammation [22,23].

While anti-inflammatory cytokines were not measured in this study, a CARS-associated down-regulation of chronic inflammation is consistent with the pattern of change in CRP observed following ELS treatment. At 12 weeks, CRP levels are increased despite normal circulating leukocyte counts. Thereafter, a progressive decline out to 48 weeks is observed. This was not due to a large decline in CPR in small subset of patients, but rather represents a uniform decrease in CRP among the cohort population at large. Eight (8) of 16 patients initially observed to have CRP values in the "high risk" category (i.e. $\geq 3 \mathrm{mg} / \mathrm{L}$ ) experienced declines into the healthy normal range by week 48. Eight additional patients initially in the "high risk" group experienced declines in CRP that did not result in a drop below 3 $\mathrm{mg} / \mathrm{L}$. Only 1 of 29 patients experienced an increase in CRP from $<3$ $\mathrm{mg} / \mathrm{L}$ to $\geq 3 \mathrm{mg} / \mathrm{L}$ at 48 weeks following ELS treatment.

Results presented in Figure 4 suggest that ELS therapy is associated with better survival out to 3 years post treatment than predicted based on baseline BODE Index scores. While comparisons of this type must be validated prospectively, they are consistent with the emerging belief that, similar to $\mathrm{CHD}$, chronic inflammation may be an important determinant of long term outcomes in emphysema, and effective treatments that address both the pathobiology and pathophysiology of emphysema could substantially impact long term outcomes in this population.

Although lack of a control group represents an important limitation in this study, the natural history of advanced emphysema has been previously well documented6. Among patients with GOLD Group D emphysema receiving optimal medical therapy, CRP has been shown to increase over time. Thus the declines in CRP observed cannot be accounted for simply by changes in medical management associated with clinical trial participation. Nevertheless, these results await future validation in randomized controlled trials. If confirmed, these data would suggest that peri-procedural inflammation associated with certain types of endoscopic lung volume reduction may have long-term benefits by altering the immune status of patient's which advanced emphysema and active chronic inflammation.

\section{Conflict of Interest}

Prof. Kramer, Dr. Fruchter, Prof Herth, Dr. Eberhardt, Dr. Valipour, Dr. Assadi, Dr. Kempa, Dr. Bonnet, Dr. Maeder, and Dr. Stanzel have no conflicts of interest related to the data presented in this manuscript. Dr. Ingenito is Chief Scientific Officer of Aeris Therapeutics, the study sponsor and manufacturer of Emphysematous Lung Sealant.

\section{Source of Support}

Aeris Therapeutics, 10K Gill Street, Woburn, MA 01801, USA. 
Citation: Kramer MR, Fruchter O, Eberhardt R, Valipour A, Assadi S, et al. (2013) Emphysematous Lung Sealant Therapy Attenuates the Chronic Inflammatory State of Advanced Emphysema. J Pulm Respir Med 3: 163. doi:10.4172/2161-105X.1000163

\section{References}

1. Pinto-Plata V, Casanova C, Müllerova H, de Torres JP, Corado H, et al. (2012) Inflammatory and repair serum biomarker pattern: association to clinical outcomes in COPD. Respir Res 13: 71.

2. Celli BR, Barnes PJ (2007) Exacerbations of chronic obstructive pulmonary disease. Eur Respir J 29: 1224-1238.

3. Celli BR, Locantore N, Yates J, Tal-Singer R, Miller BE, et al. (2012) Inflammatory biomarkers improve clinical prediction of mortality in chronic obstructive pulmonary disease. Am J Respir Crit Care Med 185: 1065-1072.

4. Pinto-Plata VM, Livnat G, Girish M, Cabral H, Masdin P, et al. (2007) Systemic cytokines, clinical and physiological changes in patients hospitalized for exacerbation of COPD. Chest 131: 37-43.

5. Celli BR, Thomas NE, Anderson JA, Ferguson GT, Jenkins CR, et al. (2008) Effect of pharmacotherapy on rate of decline of lung function in chronic obstructive pulmonary disease: results from the TORCH study. Am J Respir Crit Care Med 178: 332-338.

6. Mineo D, Ambrogi V, Cufari ME, Gambardella S, Pignotti L, et al. (2010) Variations of inflammatory mediators and alpha1-antitrypsin levels after lung volume reduction surgery for emphysema. Am J Respir Crit Care Med 181: 806-814

7. Mineo D, Ambrogi V, Frasca L, Cufari ME, Pompeo E, et al. (2008) Effects of lung volume reduction surgery for emphysema on glycolipidic hormones. Chest 134: 30-37.

8. Fishman A, Martinez F, Naunheim K, Piantadosi S, Wise R, et al. (2003) A randomized trial comparing lung-volume-reduction surgery with medical therapy for severe emphysema. N Engl J Med 348: 2059-2073.

9. Criner GJ, Sternberg AL (2008) National Emphysema Treatment Trial: the major outcomes of lung volume reduction surgery in severe emphysema. Proc Am Thorac Soc 5: 393-405

10. Dahl M, Nordestgaard BG (2009) Markers of early disease and prognosis in COPD. Int J Chron Obstruct Pulmon Dis 4: 157-167.

11. Herth FJ, Eberhardt R, Ingenito EP, Gompelmann D (2011) Assessment of a novel lung sealant for performing endoscopic volume reduction therapy in patients with advanced emphysema. Expert Rev Med Devices 8: 307-312.

12. Barreiro E, del Puerto-Nevado L, Puig-Vilanova E, Pérez-Rial S, Sánchez F, et al. (2012) Cigarette smoke-induced oxidative stress in skeletal muscles of mice. Respir Physiol Neurobiol 182: 9-17.

13. Churg A, Wang RD, Tai H, Wang X, Xie C, et al. (2003) Macrophage metalloelastase mediates acute cigarette smoke-induced inflammation via tumor necrosis factor-alpha release. Am J Respir Crit Care Med 167: 10831089.

14. Kim V, Criner GJ, Abdallah HY, Gaughan JP, Furukawa S, et al. (2005) Small airway morphometry and improvement in pulmonary function after lung volume reduction surgery. Am J Respir Crit Care Med 171: 40-47.

15. Kim V, Rogers TJ, Criner GJ (2008) New concepts in the pathobiology of chronic obstructive pulmonary disease. Proc Am Thorac Soc 5: 478-485

16. Hogg JC, Chu FS, Tan WC, Sin DD, Patel SA, et al. (2007) Survival after lung volume reduction in chronic obstructive pulmonary disease: insights from small airway pathology. Am J Respir Crit Care Med 176: 454-459.

17. McDonough JE, Yuan R, Suzuki M, Seyednejad N, Elliott WM, et al. (2011) Small-airway obstruction and emphysema in chronic obstructive pulmonary disease. N Engl J Med 365: 1567-1575.

18. Arant CB, Wessel TR, Ridker PM, Olson MB, Reis SE, et al. (2009) Multimarke approach predicts adverse cardiovascular events in women evaluated for suspected ischemia: results from the National Heart, Lung, and Blood Institutesponsored Women's Ischemia Syndrome Evaluation. Clin Cardiol 32: 244-250.

19. Johnson BD, Kip KE, Marroquin OC, Ridker PM, Kelsey SF, et al. (2004) Serum amyloid $A$ as a predictor of coronary artery disease and cardiovascular outcome in women: the National Heart, Lung, and Blood Institute-Sponsored Women's Ischemia Syndrome Evaluation (WISE). Circulation 109: 726-732.

20. Ridker PM (2004) High-sensitivity C-reactive protein, inflammation, and cardiovascular risk: from concept to clinical practice to clinical benefit. Am Heart J 148: S19-26.

21. Herth FJ, Gompelmann D, Stanzel F, Bonnet R, Behr J, et al. (2011) Treatment of advanced emphysema with emphysematous lung sealant (AeriSeal囚).
Respiration 82: 36-45.

22. Adib-Conquy M, Cavaillon JM (2009) Compensatory anti-inflammatory response syndrome. Thromb Haemost 101: 36-47.

23. Simmons EM, Himmelfarb J, Sezer MT, Chertow GM, Mehta RL, et al. (2004) Plasma cytokine levels predict mortality in patients with acute renal failure. Kidney Int 65: 1357-1365. 